\title{
LOCALIZATION AND REGIONAL ASPECTS OF GAME PRODUCTION - A RESEARCH OVERVIEW
}

\author{
Marcus Toftedahl \\ University of Skövde, Division of Game Development, \\ Högskolevägen 2, Skövde, Sweden
}

\begin{abstract}
This paper focus on how localization is addressed in research on game development processes. The research aim is to get an understanding of how localization, game localization and its subsets of content and cultural issues, linguistic issues and technical issues are addressed and discussed in game development research. This has been done through a literature study, where 488 papers regarding game development processes have been scrutinized in order to identify game localization related research. The results shows that localization is rarely addressed in game development research, but regional aspects and relevant concepts related to game localization can be found.
\end{abstract}

\section{KEYWORDS}

Localization, Game Development, Regional Aspects, Game Development, Creative Industries, Software Development

\section{INTRODUCTION}

Localization is the "process of modifying products or services to account for differences in a distinct market", a definition provided by the (now defunct) Localization Industry Standards Association (LISA) (Fry \& Lommel, 2003, p. 13). Further, localization is often divided into three categories that the process of localization needs to address; linguistic issues, content and cultural issues and technical issues. In software development the need of localization has its origins with the advent of office software such as word processors and spreadsheet software in the late 1970's (O'Hagan \& Mangiron, 2013). When the software was distributed outside the market of origin, alterations needed to be made according to differences in language, but also in other areas such as formatting conventions including the format of dates, numbering and currency. O'Hagan and Mangiron (2013) describe this as a major shift in mindset when producing software for a global market, where the software developers began to not only localize a specific product but change development practices towards a more localization friendly development cycle. A product that is produced in one part of the world can immediately be available for purchase in another. This is a challenge for all product development, making localization an important factor of the production process.

In a previously conducted literature review containing 488 research articles on game development and management of creativity, it was identified that the vast majority of articles relating to game development took little to no consideration of regional aspects in their research approaches (Engström, Marklund, Backlund, \& Toftedahl, 2018). Further, $84 \%$ of the articles from the study did not mention regional aspects, and only $2.6 \%$ had regional aspects as main focus. It should be noted that regional aspects in this case refers to a broad area of aspects, including practices, data from specific regions, location of companies etc. - i.e. not strictly localization related content.

This paper is written with the aim to survey how (if?) localization is addressed in research on game development processes. The research aim is to get an understanding of how localization, game localization and its subsets of content and cultural issues, linguistic issues and technical issues are addressed and discussed in game development research. 


\section{PREVIOUS WORK}

This research has been conducted within the overarching research project Game Hub Scandinavia (GHS), focusing on exploring the current state of game development practice from an academic and industry perspective. Examples of other topics explored within GHS, thus in some ways related to this paper, are: localization in indie game development studios (Toftedahl, Backlund, \& Engström, 2018); game engines and production tools (Toftedahl \& Engström, 2019); and the two literature studies described below (Engström et al., 2018; Marklund, Engström, Hellkvist, \& Backlund, 2019).

While the aim of this article is to explore how game localization is addressed in research articles regarding the craft and processes of game development, this research is based on methods, data and results from the larger literature study conducted by Engström et al. (2018). In the study, a large number of research articles regarding game development and creative industries were identified and scrutinized, focusing on how contemporary research takes the duality of video game development from a software development and creative production perspective into account. In the initial literature review 2278 publications were included, subsequently reduced to a set of 488 papers. The selection process involved identifying only papers written in English and published between 2006 and 2016. To briefly summarize the literature selection process, a series of keywords related to game development or creative- and cultural industries in conjunction with development processes were used in several wide-reaching databases (Scopus, Springer, ACM, and DiGRA digital archive) to generate the initial set of 2278 publications. Through title and abstract analysis, this set was then reduced to 488 papers that were reviewed and coded. During the coding process three particular parameters relevant for the study were included: research quality evaluation; identification whether a paper was based on empirical data from the games industry; and, if regional aspects were addressed. The details of the fundamental method of the study are described in detail in Engström et al. (2018).

A continuation of the literature review project was conducted by Marklund et al. (2019), going more in-depth on one of the research questions used in the initial literature review. Using the 488 papers from the initial literature review, the paper by Marklund et al. (2019) focuses solely on papers with empirically grounded research based on data informed by game developers or game development studios. In this paper, a subset of 48 of the initial 488 papers was used. All included papers were fulfilling the following criteria: they contain empirical data from industry practitioners (i.e. not development conducted in an academic setting); they display a high quality of research (i.e. having a clearly stated research question, method description and results); and, they are relevant to our understanding to the practices of game development.

\section{METHOD}

In order to understand how game localization and regional aspects of game development have been discussed in previous literature a review using the papers identified in Engström et al. (2018) and Marklund et al. (2019) has been carried out. Since the previous studies using the dataset have had different aims, an approach to include as many of the previously identified papers has been made. To accomplish this, two passes of review have been made.

Firstly, a simple full text search of the total of 488 papers included in the original literature review by Engström et al. (2018) was conducted. To catch papers relevant for this study (i.e. game localization) a word search for "locali*" and "translat*" was made on the set of papers to include variations of localization and translation (i.e. different spellings, capitalization etc.). With the specific key word search, articles mentioning localization and related concepts should appear search query. The query resulted in 61 articles in total. Upon closer inspection only 16 articles were about the practice of localization, the other mentioned localization in other contexts and meanings such where the majority was about physical locations and re-localization and localization economies versus urbanization economies as a part of emerging "creative cities and regions". The same trend was seen in relation to the "translat*" search, where the term translation in relation to mathematics was found.

Since the corpus of 488 papers included research from software engineering and creative software development in general as well as game development, a reading of titles and abstracts was conducted to find the papers that had a specific focus on game development. Five papers were removed in this pass, not mentioning game development. In the end 11 papers regarding game development was found to cover some aspects of localization. 
Secondly, a thematic analysis of the 48 papers from the study by Marklund et al. (2019) and the papers identified in the previously described localization keyword search was conducted. Thematic analysis is described by Braun and Clarke (2006, p. 6) as:

"[...] a method for identifying, analyzing, and reporting patterns (themes) within data. It minimally organizes and describes your data set in (rich) detail. However, it also often goes further than this, and interprets various aspects of the research topic"

One paper identified in the keyword search was already included in the set of 48 papers, thus 10 additional papers were added to a specific group to be able to separate the two groups of papers. The papers included from the study by Marklund et al. (2019) are also a subset of the corpus of papers used in Engström et al. (2018), with a focus on game development with data from game developers and game development studios. This subset of papers do therefore already have a specific scope regarding game development, thus making a more thorough analysis regarding localization is plausible to catch game localization descriptions where other phrasings (than localization) are being used.

As this review aims to identify game localization related topics in a corpus of articles previously identified to describe game development, a continuation of the thematic analysis done by Marklund et al. (2019) has been carried out. The coding process was conducted using the MAXQDA qualitative analysis software program allowing both thematic coding and free text commenting of large data sets. The MAXQDA software can after the coding present compilations of the coded segments and comments for easier analyses. The thematic analysis process was divided into three main phases; a preparation phase, a content processing and coding phase, and an analysis phase. In more detail, the first phase involved familiarization with the corpus of papers and identifying relevant codes related to the aim of this paper. The coding categories from the study by Marklund et al. (2019) was kept intact in order to be able to cross-reference game localization aspects with the previously identified game development related information. One code from the previous analysis was left unaltered and to be included in the game localization coding; the identification of "Region of business" (i.e. data gathered from a game developer in a specific country). The codes chosen for the localization related material was mainly based on aspects derived from the three categories of localization (i.e. linguistic, cultural and technical issues) (O'Hagan \& Mangiron, 2013) and the Global product development cycle (Fry \& Lommel, 2003). The final codes used for the localization related aspects of game development were:

- Regional aspects - over-arching category, divided into following sub-categories:

- Data from specific region/s - results based on data from a specific, named region/country.

- Development and localization - development processes with direct relation to localization.

- Localization - paper mentions localization specifically.

- Technical issues - data or results related to technical issues of localization.

- Linguistic issues - data or results related to linguistic issues of localization.

- Cultural issues - data or results related to cultural issues of localization.

- Business strategies - specific business strategies related to different regions.

- Globalness of the game industry - statements related to the global nature of the game industry.

The content processing and coding phase involved scrutinizing the papers and using MAXQDA to apply the corresponding codes to the papers. The complete set of papers was analyzed by reading through them and marking text segments with codes from the established code categories. The third and final phase, the analysis phase, involved searching for themes in the compiled and coded material for analysis. The themes and trends found in the coded material were then grouped to make the themes easy to describe and present.

\section{RESULTS}

The literature study shows that from a corpus of 488 papers regarding game development and management of creativity very few are addressing game localization aspects of game development. The keyword search conducted on the 488 papers only gave 16 results regarding localization, and 11 of them were papers focusing on game development in one way or another. Using the dataset from the study Marklund et al. (2019) containing 48 papers, 27 papers mentions regional aspects in general. 
The set of papers specific for this study hence consisted of 37 papers in total. The 37 papers are combination of the 11 papers from the keyword search and the 27 papers mentioning regional aspects from the study by Marklund et al. (2019), with one paper overlapping the two sets. Table 1 shows a quantitative overview of the occurrences of the codes, where number of documents containing the specific code category is shown as well as the total number of occurrences.

Table 1. Quantitative overview of the coded documents

\begin{tabular}{|r|c|c|}
\hline $\begin{array}{c}\text { Code category (sub-categories } \\
\text { of Regional aspects) }\end{array}$ & $\begin{array}{c}\text { Number of documents } \\
\text { containing code }\end{array}$ & $\begin{array}{r}\text { Total number } \\
\text { of occurrences }\end{array}$ \\
\hline Data from specific region & 23 & 23 \\
\hline Globalness of game industry & 17 & 24 \\
\hline Localization & 11 & 14 \\
\hline Business strategies & 7 & 12 \\
\hline Development and localization & 7 & 8 \\
\hline Cultural (aspects of loc.) & 4 & 2 \\
\hline Technical (aspects of loc.) & 2 & 2 \\
\hline Linguistic (aspects of loc.) & 2 & 25 \\
\hline
\end{tabular}

The most frequent element of regional aspects found in the papers is research based on data from a specific region. This is where it is explicitly stated in the paper that the data is gathered from a specific region, i.e. often found in the method description of the paper. The most common region regarding this aspect is Finland, with 9 papers based on data from Finnish game developers. The second most frequent region of data collection is USA with 3 papers, followed by Canada with 2 papers. Table 2 shows a breakdown of the data from specific regions in more detail.

Table 2. Papers with data from specific regions

\begin{tabular}{|c|c|c|c|c|c|c|c|c|c|c|c|c|c|}
\hline Paper/Country & 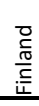 & گ్ & $\begin{array}{l}\frac{\pi}{0} \\
\pi \\
\frac{\pi}{\pi} \\
\tilde{J} \\
\end{array}$ & $\begin{array}{l}\stackrel{0}{\frac{C}{二}} \\
\text { U. } \\
\end{array}$ & 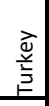 & $\begin{array}{l}\text { đ̊ } \\
\stackrel{0}{0} \\
\underline{1}\end{array}$ & 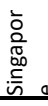 & 兰 & $\begin{array}{l}\bar{Z} \\
\frac{1}{0} \\
\frac{\pi}{0} \\
\end{array}$ & 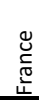 & 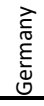 & 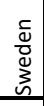 & 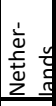 \\
\hline Hagen (2004) & & & & & & & & & & & & $\mathbf{x}$ & \\
\hline Lee, Lee, Cho, Song, and Rhew (2006) & & & & & & $\mathbf{x}$ & & & & & & & \\
\hline Myllärniemi, Raatikainen, and Männistö (2006) & $\mathbf{x}$ & & & & & & & & & & & & \\
\hline Tschang and Szczypula (2006) & & $\mathbf{x}$ & & & & & & & & & & & \\
\hline van de Weerd, de Weerd, and Brinkkemper (2007) & & & & & & & & & & & & & $\mathbf{x}$ \\
\hline Stacey and Nandhakumar (2008) & & & & & & & $\mathbf{x}$ & & & & & & \\
\hline Kultima (2010) & $\mathbf{x}$ & & & & & & & & & & & & \\
\hline Binark and Bayraktutan (2012) & & & & & $\mathbf{x}$ & & & & & & & & \\
\hline Legault and Ouellet (2012) & & & $\mathbf{x}$ & & & & & & & & & & \\
\hline Wimmer and Sitnikova (2012) & & & & & & & & & & & $\mathbf{x}$ & & \\
\hline Chung and Fung (2013) & & & & $\mathbf{x}$ & & & & & & & & & \\
\hline Hodgson and Briand (2013) & & & $\mathbf{x}$ & & & & & & & & & & \\
\hline Kasurinen, Laine, and Smolander (2013) & $\mathbf{x}$ & & & & & & & & & & & & \\
\hline Kasurinen, Strandén, and Smolander (2013) & $\mathbf{x}$ & & & & & & & & & & & & \\
\hline Koutonen and Leppänen (2013) & $\mathbf{x}$ & & & & & & & & & & & & \\
\hline Lê, Massé, and Paris (2013) & & & & & & & & & & $\mathbf{x}$ & & & \\
\hline Kasurinen, Maglyas, and Smolander (2014) & $\mathbf{x}$ & & & & & & & & & & & & \\
\hline Kasurinen and Smolander (2014) & $\mathbf{x}$ & & & & & & & & & & & & \\
\hline Murphy-Hill, Zimmermann, and Nagappan (2014) & & $\mathbf{x}$ & & & & & & & & & & & \\
\hline Musial, Kauppinen, and Puhakka (2016) & $\mathbf{x}$ & & & & & & & $\mathbf{x}$ & $\mathbf{x}$ & & & & \\
\hline Nelson and Palumbo (2014) & & $\mathbf{x}$ & & & & & & & & & & & \\
\hline Vanhala and Kasurinen (2014) & $\mathbf{x}$ & & & & & & & & & & & & \\
\hline
\end{tabular}


Regarding the globalness of the game industry, 17 papers mentioning the global nature of the game industry were found. This is a wide category, where both remarks of how the game industry is a global business and more in-depth analyses regarding this are made. This category often has a business perspective, thus cross-coded with the business strategy code. One example of this is from Tschang (2009):

"Hollywood movies have a world-wide following, no less in Asia than in other regions. The US-made online game World of Warcraft (WOW) has become a worldwide phenomenon, with a large proportion of its revenues coming from China."

Chung and Fung (2013, p. 248) presents another perspective, regarding how the globalness of the game industry is reflected in business decisions, focusing on local and regional business practices:

"The Chinese online gaming companies have reached a stage where locally developed games can compete with quality foreign games from Korea and the United States. Some Chinese game companies now export their products to nearby Asian countries, competing with regional markets traditionally dominated by Korean game companies. While the state-market framework has protected local game companies within the Chinese market, the real competition and arena for expansion takes place outside the country, in the world market."

While both Hollywood movies and big blockbuster games such as World of Warcraft are often used as examples of the global game industry, there are examples where small-scale companies/developers and their perspectives are considered as well. With a Finnish perspective, Vanhala and Kasurinen (2014, p. 84) mention the importance of having a global strategy for small companies:

\footnotetext{
"We studied six computer game start-ups in Finland. This means that the sample size was small and homogeneous. However, all the companies were aiming at the international markets with their products, the companies covered different release platforms and genres, and were developing games as their main source of income, so the companies did have variance and were representative organizations of the games industry."
}

Even though overarching business strategies in the global game industry is an important topic, there are few articles mentioning how to handle the global nature of the game industry from a development perspective. Only 7 articles have been identified to discuss the development aspect of localization in one way or another. In an article by van de Weerd et al. (2007) with the approach to construct a reference method for game production, localization is lifted as a specific task in game production. The reference method for game production is derived from four game production methods, constructing one super method containing activities and concepts from the four included production methods. van de Weerd et al. (2007) use three methods described in the following three game production books: Game Development and Production (Bethke, 2003); Introduction to Game Development (Rabin, 2005); and, The Game Production Handbook (Chandler, 2009). The forth method in the study is a proprietary method used at a game development company. van de Weerd et al. (2007, p. 316) present one version of a game production process, quoting Chandler (2009):

"Chandler describes in 'The Game Production Handbook' four main processes in the game production cycle. These are Pre-Production, Production, Testing and Post-Production. The pre-production phase encompasses the definition of the game concept, the definition of the game requirements and the definition of the game plan. After pre-production there is production, in which builds of the game are created and localized."

Further, van de Weerd et al. (2007, p. 319) gives an overview of the proprietary game production method included in the study, based on the processes of a game development company called Zylom:

"The game production process as it is carried out at Zylom is divided into four phases, namely Design, Development, Quality assurance and Localization. The method process consists of 11 activities, 59 activities, and 60 concepts."

Regarding localization in production, van de Weerd et al. (2007) emphasizes the lack of in-depth descriptions of the localization process. In the development process descriptions from Introduction to Game Development (Rabin, 2005) it is noted by van de Weerd et al. (2007, p. 322) that detailed descriptions of 
hiring staff members and performing market and sales related activities is included, but localization is not described using the same detail:

"The ITGD method is the only one to address in detail the hiring of staff members as part of the game production process and performing marketing and sales related activities. Activities are not only management specific; also the actual game development process is covered in quite some detail. However, the localization of finished games is described very briefly and is therefore too basic to be really useful."

As a contrast to the brief description in Introduction to Game Development, van de Weerd et al. (2007) notes that The Game Production Handbook has a very detailed description of the localization process:

"The TGPH method excels when it comes to the localization process and the finalization of the game production project. The localization steps and the definition and creation of closing kits are thoroughly discussed. Finally, the part of the project in which code is released is also well explained in TGPH compared to other methods."

As a conclusion, van de Weerd et al. (2007) have included localization in their amalgam of the four game development processes. Localization is in their proposed reference method placed in the post-production phase, in conjunction with quality assurance. The conclusion that localization is an activity carried out in the later stages of development are mirrored in other papers as well; Bernhaupt (2015) mentions localization as a part of the later development stage, Zoeller (2013) identifies that quality assurance managers and localization producers wants to know where in the game foreign language testers encounters problem, and Deuze, Martin, and Allen (2007) explicitly mention localization as an outsourcing activity.

Regarding localization and its components of cultural, technical and linguistic issues, few papers mention any of the specifics. Regarding technical issues, Chung and Fung (2013) mention the development of broadband access in Asian countries and connects this to the possibility of growing e-services. Alves, Ramalho, and Damasceno (2007, p. 276) connect growing technical infrastructures as a possibility to expand into new markets:
"On the one side, the game must be available in several languages, adhere to particular regional requirements and developers have to orchestrate complex distribution channels. On the other side, the increasing number of mobile phone subscribers, especially in developing countries, represents a huge unexplored consumer market."

The quote from Alves et al. (2007, p. 276) above is also one of few articles mentioning linguistic issues, even briefly. Linguistic issues, regarding that the game must be available in a language that the player can understand is not a topic discussed in detail in any of the articles included in this study.

Cultural issues are mentioned in some articles from two main perspectives: local regulations and knowledge of local references. Both Chung and Fung (2013) and Bernhaupt (2015) mentions how the knowledge of local rules and regulations must be taken into account. Bernhaupt $(2015$, p. 5) means that localization is an important phase for games where adaptations to the game content must be made:

"Localization: an important phase for games that will be delivered to different markets (countries) is the localization phase. In this phase game-play can be adjusted to suit the tastes of the market, to allow language translation and modifications due to local regulatory authorities."

Chung and Fung (2013) gives an example where the complex nature of the local Chinese regulations and censorship of games are described. The description gives an overview of the relations between a number of Chinese governmental agencies handling the authorization and censorship of games in China. In general, one agency has the power allow or ban any media publication and another agency reviews the content of games based on certain regulations involving protecting minors from disturbing content etc. Thus, the approval process is long and complicated and Chung and Fung (2013, p. 240) concludes:

"Our talks with these government agencies and game companies revealed that, before a foreign game is released in the Chinese market, game companies have to receive dual approval from these two agencies."

Callele, Neufeld, and Schneider (2006, p. 44) mention cultural conditioning in localized products as an area important to take into consideration: 


\begin{abstract}
"Emotional requirements can require localization efforts. International audiences (and members of international teams) might interpret the same symbols and events differently. Test your scenarios on your target markets to ensure that they don't elicit inadvertent interpretations. Perhaps the most cited example is the color red, which in North America means danger, whereas in China, red means good fortune."
\end{abstract}

Also, local traditional stories, myths and legends that are common in one region but incomprehensible in another is an aspect that needs to be addressed. Tschang (2009, p. 38) gives one example of this:

One especially popular theme has been the Three Kingdoms period of Chinese history where states were at war. On the other hand, while Chinese culture may sell well in countries that neighbour China (e.g. Chinese online games being popular in Vietnam and Taiwan), this does not necessarily carry over to the global market."

\title{
5. CONCLUSIONS
}

Game localization from a development perspective has been identified as an area where more research is needed. It was identified already in the previous studies (Engström et al., 2018; Marklund et al., 2019) that the vast majority of articles relating to game development have taken little or no consideration of regional aspects in their research approaches and regional aspects that might affect game development are seldom discussed. The main conclusion from this literature review is building further upon that statement. Localization is rarely discussed in game development research, and if it is discussed it is briefly mentioned.

Even though the set of articles used in this paper can be questioned - localization or regional aspects was not an inclusion criteria in the original data collection phase - it is rather peculiar that the process of localization is only mentioned briefly. The set of papers this study is based on includes papers published until 2016, an obvious flaw in a fast moving field. A quick search in the databases used in the original literature review (i.e. Scopus, Springer, ACM, and DiGRA digital archive) using combinations of game, development and localization published between 2017-2020 in the search query, revealed only one article focusing on the specific topic of this paper; the aforementioned article on indie game localization by Toftedahl et al. (2018) also from the GHS project.

One aspect of the lack of research of localization with a production perspective is the difficulty of obtaining access to developers and development material. Bernal-Merino (2014) and O'Hagan and Mangiron (2013) have identified this, where confidentiality issues and non-disclosure agreements makes it difficult to get access to production settings. Even though research on localization is limited, there are research done highly relevant for the understanding of localization as a process in game development. Going beyond the localization terminology, aspects related to the process can be found. For example, the localization subcategories of cultural-, technical-, and linguistic issues can be found if looked for as the results from this paper shows. Thanks to the broad scope of the papers included in the dataset, these more specific areas could be found when scrutinized. One example is the paper by Chung and Fung (2013) where the commercialization of Chinese online gaming is the main topic, but both technical and cultural aspects of this are addressed. While not framed explicitly as a localization paper, it covers aspects very relevant for the understanding of how work within the Chinese market.

As a contrast to this literature study, where localization is scrutinized from a game development research perspective, a literature study by Mangiron (2017) has conducted a translation studies perspective of game localization. Mangiron presents a historical overview of the state of game localization research, identifying a number of common research topics and methods. Most research from this perspective are conducted as descriptive studies within translation, mainly with qualitative approaches. Mangiron (2017) also highlights the interdisciplinary nature of the topic, where research angles from translation studies, game studies, media studies and culture studies have been identified. Research on game localization have had a strong focus on cases involving console games, and virtually no attempts has been identified regarding the growing field of mobile games. A strong dominance of Japanese games has also been identified. From a development perspective Mangiron (2017) highlights a study by O'Hagan and Chandler (2016) where it is stated that:

\footnotetext{
"Game localization has been largely ignored in game studies and constitutes a relatively new area of research in translation studies".
} 
Even though more research on game localization is needed, from several perspectives, the approaches made contains vital information for future research approaches. The approach taken by van de Weerd et al. (2007) where a number of game development process descriptions have been combined into a super process could easily be adapted to a game localization process description, taking information from both game development and translation studies. Further, to scrutinize general game development process descriptions from both academic and industry sources could be a way of identifying questions (and answers) regarding the complexities of game localization and its connection to current game development practices.

\section{ACKNOWLEDGEMENT}

This research is funded by the EU Interreg ÖKS project Game Hub Scandinavia 2.0.

\section{REFERENCES}

Alves, C., Ramalho, G., \& Damasceno, A. (2007). Challenges in requirements engineering for mobile games development: The meantime case study. Paper presented at the 15th IEEE International Requirements Engineering Conference (RE 2007).

Bernal-Merino, M. Á. (2014). Translation and localisation in video games: making entertainment software global: Routledge.

Bernhaupt, R. (2015). User experience evaluation methods in the games development life cycle Game user experience evaluation (pp. 1-8): Springer.

Bethke, E. (2003). Game development and production: Wordware Publishing, Inc.

Binark, M., \& Bayraktutan, G. (2012). A Critical Interpretation of a New "Creative Industry" in Turkey: Game Studios and the Production of a Value Chain Computer games and new media cultures (pp. 371-391): Springer.

Braun, V., \& Clarke, V. (2006). Using thematic analysis in psychology. Qualitative research in psychology, 3(2), 77-101.

Callele, D., Neufeld, E., \& Schneider, K. (2006). Emotional requirements in video games. Paper presented at the 14th IEEE International Requirements Engineering Conference (RE'06).

Chandler, H. M. (2009). The game production handbook: Jones \& Bartlett Publishers.

Chung, P., \& Fung, A. (2013). Internet development and the commercialization of online gaming in China Gaming Globally (pp. 233-250): Springer.

Deuze, M., Martin, C. B., \& Allen, C. (2007). The professional identity of gameworkers. Convergence, 13(4), 335-353.

Engström, H., Marklund, B. B., Backlund, P., \& Toftedahl, M. (2018). Game development from a software and creative product perspective: A quantitative literature review approach. Entertainment Computing, 27, 10-22.

Fry, D., \& Lommel, A. (2003). The localization industry primer.

Hagen, U. (2004). Where do game design ideas come from? Innovation and recycling in games developed in Sweden. World, 24, 2007.

Hodgson, D., \& Briand, L. (2013). Controlling the uncontrollable:'Agile'teams and illusions of autonomy in creative work. Work, employment and society, 27(2), 308-325.

Kasurinen, J., Laine, R., \& Smolander, K. (2013). How Applicable Is ISO/IEC 29110 in Game Software Development?, Berlin, Heidelberg.

Kasurinen, J., Maglyas, A., \& Smolander, K. (2014). Is requirements engineering useless in game development? Paper presented at the International Working Conference on Requirements Engineering: Foundation for Software Quality.

Kasurinen, J., \& Smolander, K. (2014). What do game developers test in their products? Paper presented at the Proceedings of the 8th ACM/IEEE International Symposium on Empirical Software Engineering and Measurement.

Kasurinen, J., Strandén, J.-P., \& Smolander, K. (2013). What do game developers expect from development and design tools? Paper presented at the Proceedings of the 17th International Conference on Evaluation and Assessment in Software Engineering.

Koutonen, J., \& Leppänen, M. (2013). How are agile methods and practices deployed in video game development? A survey into Finnish game studios. Paper presented at the International Conference on Agile Software Development.

Kultima, A. (2010). The organic nature of game ideation: game ideas arise from solitude and mature by bouncing. Paper presented at the Proceedings of the International Academic Conference on the Future of Game Design and Technology. 
Lê, P. L., Massé, D., \& Paris, T. (2013). Technological Change at the Heart of the Creative Process: Insights From the Videogame Industry. International journal of arts management, 15(2).

Lee, S. H., Lee, G. H., Cho, H. H., Song, D. H., \& Rhew, S. Y. (2006). An empirical model of the game software development processes. Paper presented at the Fourth International Conference on Software Engineering Research, Management and Applications (SERA'06).

Legault, M.-J., \& Ouellet, K. (2012). So Into It They Forget What Time It Is?: Video Game Designers and Unpaid Overtime Managing dynamic technology-oriented businesses: High-tech organizations and workplaces (pp. 82-102): IGI Global.

Mangiron, C. (2017). Research in game localisation. The Journal of Internationalization and Localization, 4(2), 74-99.

Marklund, B. B., Engström, H., Hellkvist, M., \& Backlund, P. (2019). What Empirically Based Research Tells Us About Game Development. The Computer Games Journal, 8(3-4), 179-198.

Murphy-Hill, E., Zimmermann, T., \& Nagappan, N. (2014). Cowboys, ankle sprains, and keepers of quality: How is video game development different from software development? Paper presented at the Proceedings of the 36th International Conference on Software Engineering.

Musial, M., Kauppinen, A., \& Puhakka, V. (2016). Recognised Creativity: The Influence of Process, Social Needs, and the Third Drive on Creative Individuals' Work through Social Media Social media and networking: Concepts, methodologies, tools, and applications (pp. 1249-1280): IGI Global.

Myllärniemi, V., Raatikainen, M., \& Männistö, T. (2006). Inter-organisational approach in rapid software product family development - a case study. Paper presented at the International Conference on Software Reuse.

Nelson, W. A., \& Palumbo, D. B. (2014). When design meets Hollywood: Instructional design in a production studio environment Design in Educational Technology (pp. 75-88): Springer.

O'Hagan, M., \& Mangiron, C. (2013). Game Localization: Translating for the global digital entertainment industry (Vol. 106): John Benjamins Publishing.

O’Hagan, M., \& Chandler, H. (2016). Game localization research and translation studies. Border crossings: Translation studies and other disciplines, 126, 309.

Rabin, S. (2005). Introduction to game development: Charles River Media Boston.

Stacey, P., \& Nandhakumar, J. (2008). Opening up to agile games development. Communications of the ACM, 51(12), 143-146.

Toftedahl, M., Backlund, P., \& Engström, H. (2018). Localization from an Indie Game Production Perspective: Why, When and How? Paper presented at the DiGRA '18 - Proceedings of the 2018 DiGRA International Conference: The Game is the Message, Torino, Italy.

Toftedahl, M., \& Engström, H. (2019). A Taxonomy of Game Engines and the Tools that Drive the Industry. Paper presented at the DIGRA INTERNATIONAL CONFERENCE 2019: GAME, PLAY AND THE EMERGING LUDO-MIX

Tschang, F. T. (2009). Creative industries across cultural borders: The case of video games in Asia Creative economies, creative cities (pp. 25-42): Springer.

Tschang, F. T., \& Szczypula, J. (2006). Idea creation, constructivism and evolution as key characteristics in the videogame artifact design process. European management journal, 24(4), 270-287.

van de Weerd, I., de Weerd, S., \& Brinkkemper, S. (2007). Developing a reference method for game production by method comparison. Paper presented at the Working Conference on Method Engineering.

Vanhala, E., \& Kasurinen, J. (2014). The role of business model and its elements in computer game start-ups. Paper presented at the International Conference of Software Business.

Wimmer, J., \& Sitnikova, T. (2012). The professional identity of gameworkers revisited. A qualitative inquiry on the case study of German professionals. Eludamos. Journal for Computer Game Culture, 6(1), 153-169.

Zoeller, G. (2013). Game development telemetry in production Game analytics (pp. 111-135): Springer. 